\title{
El Niño and La Niña amplitude asymmetry caused by atmospheric feedbacks
}

Claudia Frauen $^{1}$ and Dietmar Dommenget ${ }^{2}$

${ }^{1}$ IFM-GEOMAR, Leibniz Institute of Marine Sciences (at Kiel University)

${ }^{2}$ School of Mathematical Sciences, Monash University, Clayton, Australia

C. Frauen, IFM-GEOMAR, Leibniz Institute of Marine Sciences (at Kiel University), D-24105 Kiel, Germany. (cfrauen@ifm-geomar.de)

D. Dommenget, School of Mathematical Sciences, Monash University, Melbourne, Victoria, Australia.(dietmar.dommenget@sci.monash.edu.au) 


\section{Abstract}

Interannual variability of tropical Pacific sea surface temperatures (SST) has an asymmetry with stronger positive events, El Niño, and weaker negative events, La Niña, which is generally attributed to processes in the ocean. Here we present evidence from a new hybrid coupled model that the asymmetry and seasonality of El Niño can be caused by nonlinear and seasonally varying atmospheric feedbacks. The model consists of the ECHAM5 global atmospheric general circulation model (GCM) coupled to the 2-dimensional El Niño linear recharge oscillator ocean model in the tropical Pacific and a mixed layer ocean elsewhere. Despite the models simplistic and, by construction, linear representation of the ocean dynamics, it is able to simulate the main statistical features of El Niño including period, seasonality, skewness, and kurtosis. Analyses of the model show that a nonlinear relationship between zonal wind stress and SST is causing the El Niño-La Niña asymmetry. 


\section{Introduction}

The El Niño Southern Oscillation (ENSO) phenomenon is the most important source of interannual climate variability. The tropical Pacific SST is oscillating on interannual timescales with a positive anomaly during El Niño and a negative anomaly during La Niña [Philander, 1985]. The amplitude of El Niño is, however, significantly larger than the amplitude of La Niña [Burgers and Stephenson, 1999]. Further, El Niño and La Niña tend to follow a seasonal evolution, with the peak phases of the events typically happening during the end of the calendar year [Rasmusson and Carpenter, 1982].

What is causing the asymmetry of ENSO is still an open question, but most studies addressing this aspect focus on nonlinear oceanic processes [e.g. Jin et al., 2003; An and Jin, 2004; Su et al., 2010]. Jin et al. found that during the development phase of El Niño easterlies in the eastern Pacific intensify the vertical advection of anomalous warm water, what leads to an acceleration of surface warming, while during the transition to La Niña westerlies in the eastern Pacific reduce the upwelling, which leads to a slowdown of the surface cooling.

Another alternative is that nonlinear processes of the tropical atmosphere could cause the ENSO amplitude asymmetry [Hoerling et al., 1997; Kang and Kug, 2002]. Kang and Kug found indications from observations that the weaker SST anomalies during La Niña compared to El Niño may be caused by a westward shift of wind stress anomalies by $10^{\circ}$ to $15^{\circ}$. Philip and van Oldenborgh [2009] used an initially linear intermediate complexity model of the equatorial Pacific in which they introduced extra terms in the atmospheric component. Thereby they showed that the nonlinear response of mean wind stress to SST in the ENSO region has a dominant influence on the nonlinearities in SST in the ENSO cycle.

The tendency of El Niño and La Niña to peak at the end of the calendar year is one of the main features of ENSO. Seasonal factors that could cause this phase locking of ENSO are for example atmospheric heating [Philander, 1983], zonal gradients of mean SST, shallow thermocline, strong zonal winds, high 
SST [Hirst, 1986], and strong upwelling [Battisti, 1988]. But the exact physical mechanism which causes this seasonality is still discussed. Tziperman et al. [1998] suggested a seasonal amplification of Kelvin and Rossby waves by wind stress anomalies in the central Pacific could explain this seasonal evolution of ENSO.

In observations or complex climate models it is difficult to find the causes for phenomena like the ENSO skewness because of the large number of processes involved. Simple models have the advantage that they only consist of few basic physical principles and one can analyse if this basic principles are sufficient to reproduce the observed behavior. In this study we present a new hybrid coupled model, which reduces the tropical Pacific Ocean to the minimalistic low-order 2-dimensional recharge oscillator model of Burgers et al. [2005], which assumes linear oceanic feedbacks. This reduction to the minimalistic ocean model allows a more clear analysis of the atmospheric nonlinearity effects on ENSO and helps to quantify the results by the values of a few model parameters. The model results present evidence for the atmospheric cause of ENSO asymmetry and seasonality. Especially the amplitude asymmetry between eastern Pacific SST anomalies during El Niño and La Niña is investigated and possible atmospheric causes for this asymmetry are studied.

\section{Data and Models}

Observational SST data is taken from the HadISST data set for the period from 1870 to 2003 [Rayner et al., 2003]. As thermocline depth data we used the BMRC data set of the $20^{\circ}$ isotherm of Smith [1995] for the period from 1980 to 2002. The global hybrid coupled model (RECHOZ) consists of the ECHAM5 atmospheric GCM with 19 vertical levels and a horizontal spectral resolution of T31 $\left(\sim 3.75^{\circ}\right)$ [Roeckner et al., 2003]. As oceanic component outside the tropical Pacific a 19 layer single column mixed layer ocean model OZ is used [Dommenget and Latif, 2008].

In the tropical Pacific the ocean grid is replaced against a low-order 2-dimensional recharge oscillator model based on Burgers et al. [2005]. The recharge oscillator consists of two scalar equations: 


$$
\begin{aligned}
& \frac{d T}{d t}=a_{11} T+a_{12} h+\xi_{1} \\
& \frac{d h}{d t}=a_{21} T+a_{22} h+\xi_{2}
\end{aligned}
$$

with $T$ representing the NINO3 $\left(5^{\circ} \mathrm{S}-5^{\circ} \mathrm{N}, 90^{\circ} \mathrm{W}-150^{\circ} \mathrm{W}\right)$ SST anomaly, $h$ representing the mean equatorial Pacific thermocline depth anomaly and $\xi_{1}$ and $\xi_{2}$ some stochastic forcings. The values of the parameters $a_{11}, a_{12,} a_{21}$ and $a_{22}$ were chosen in accordance to the observational estimate of Burgers et al. [2005] (see Table 1). The stochastic forcings can be assumed to be the central Pacific $\left(6^{\circ} \mathrm{S}-6^{\circ} \mathrm{N}\right.$, $\left.160^{\circ} \mathrm{E}-140^{\circ} \mathrm{W}\right)$ zonal wind stress anomaly $\tau$ and the NINO3 heat flux anomaly $f$. It further has to be considered that the couplings to $T\left(a_{11}\right.$ and $\left.a_{12}\right)$ are partly caused by oceanic and atmospheric processes:

$$
a_{11}=a_{11 O}+a_{11 A} \quad(3) \quad a_{21}=a_{21 O}+a_{21 A}
$$

We assume that the atmospheric coupling to $T\left(a_{11 A} T\right.$ and $\left.a_{21 A} T\right)$ is actually a coupling to $\tau$ and $f$ :

$$
a_{11 A}=c_{\tau A} r_{T \tau}+c_{f A} r_{T f} \quad(5) \quad a_{21 A}=c_{\tau O} r_{T \tau}+c_{f O} r_{T f}
$$

The linear regressions of $\tau$ on $T, r_{T \tau}$, and $f$ on $T, r_{T f}$, were estimated from a 500yrs long uncoupled reference run, in which a harmonic oscillating SST anomaly with the pattern of the first empirical orthogonal function (EOF) of the tropical Pacific observed SST and an oscillation period of 4 years is put into the tropical Pacific (see Table 1). Thus $\tau$ and $f$ both have a part proportional to $T$ and an independent part representing the stochastic forcings $\xi_{1}$ and $\xi_{2}$.

The parameters $c_{\tau A}, c_{f A}, c_{\tau O}$, and $c_{f O}$ need to be constrained further: Following Jin [1997] we assume no heat flux forcing for $h$, thus $c_{f O}=0$; further we assume that the atmospheric heat flux is integrated by the heat capacity of the mixed layer, $m c$, with an estimated depth of $80 \mathrm{~m}$, which defines $c_{f A}$. The parameters $c_{\tau A}$ and $c_{\tau O}$ are not well constrained by neither Jin [1997] nor Burgers et al. [2005]. To 
estimate these parameters for the ECHAM5 atmosphere model we tested the RECHOZ model with a range of parameter values for $c_{\tau A}, \mathrm{c}_{\tau \mathrm{O}}$ and $m c$. For the values in Table 1 the statistics of $T$ and $h$ in the RECHOZ model are closest to those observed. So the resulting RECHOZ model equations are:

$$
\begin{aligned}
& \frac{d T}{d t}=a_{11 O} T+a_{12} h+c_{\tau A} \tau+\frac{1}{m c} f \\
& \frac{d h}{d t}=a_{21 O} T+a_{22} h+c_{\tau O} \tau
\end{aligned}
$$

Note, that the tendencies of $h$ are depending on T, while following Jin [1997] one could replace the $T$ term with a term proportional to $\tau$. We tested such a model coupled to ECHAM5, but could not find a parameter space in which realistic amplitude and oscillation of $T$ variability would occur.

To get from this one-dimensional model temperature anomaly $T$ in eq. [7] to basinwide temperatures, the resulting new temperature anomaly is multiplied with the pattern of the first EOF of the observed SST over the tropical Pacific domain and observed tropical Pacific seasonal mean SST values are added when coupled to the ECHAM5 atmosphere model. The recharge oscillator ocean model in eqs. [7 and 8] is also forced with white noise forcings (referred to as REOSC-MC) as a Monte Carlo reference model to the RECHOZ GCM. Therefore $\tau$ and $f$ are expressed in terms of $T$ using the regression coefficients mentioned above. The REOSC-MC model was integrated for 10.000 years.

In Summary, we are analyzing a hybrid coupled model RECHOZ, which has linear feedbacks in the low-order 2-dimensional ocean model and potentially nonlinear and seasonally varying feedbacks in the complex GCM atmosphere forcings $\tau$ and $f$. The parameters of the model were partly motivated by observational estimates and partly by approximations with the coupled and uncoupled ECHAM model to fit to observed NINO3 SST statistics, which will potentially give some uncertainties in the model parameters. This model is compared against the low-order 2-dimensional conceptual REOSC-MC model, which is linear in all feedbacks and forced by white noise. 


\section{Model results}

The RECHOZ model was integrated for 500 years. It simulates ENSO with very realistic statistical properties of the NINO3 SST anomalies, despite it only uses an extremely minimalistic, 2-dimensional representation of ENSO (Fig. 1). In particular the seasonality and nonlinearity of the NINO3 SST amplitudes are simulated quite realistically. The power spectrum of NINO3 SST anomalies is more regular than observed, which may be due to the simple character of the model, and has a peak between periods of about 1.5 and 4 years, which is slightly shorter than in observations, but in good agreement with other coupled GCMs. The standard deviation of the models thermocline depth anomalies is with $5.4 \mathrm{~m}$ slightly smaller than in observations $(7.5 \mathrm{~m}$ for the period from 1980 to 2002$)$ and the skewness of the models thermocline $(-1.2)$ is comparable with observations $(-0.7)$, too. The cross correlation between $T$ and $h$ (Fig. 1c), with $h$ leading the evolution of $T$ by a few month, is in good agreement with observations (as, for instance, shown in Fig. 3a of Jansen et al. [2009]).

The 99\% confidence level of the Kolmogorov-Smirnov test for a normal distribution is clearly passed (by a factor of two) for the RECHOZ SST distribution, quantifying the models non-normality. In contrast, the REOSC-MC model statistic is well within the Kolmogorov-Smirnov test distribution for a normal distribution, highlighting that the positive skewness and kurtosis in the RECHOZ model have to be caused by atmospheric forcings. This result holds also if the analysis is repeated for each season individually, to avoid seasonality effects.

\section{Atmospheric nonlinearity and seasonality}

The results of the RECHOZ model simulation indicate that atmospheric nonlinearities are responsible for the asymmetry between eastern Pacific SST anomalies during El Niño and La Niña. One possible mechanism is a nonlinear relationship between zonal wind stress anomalies and SST anomalies. Kang and Kug [2002] showed that the relatively weak SST anomalies during La Niña compared to those of 
El Niño are related to a westward shift of zonal wind stress anomalies. Composites of the zonal wind stress anomalies for El Niño and La Niña years in the RECHOZ simulation indicate a similar shift of zonal wind stress anomalies as found in observations by Kang and Kug [2002] (Fig. 2a and 2b). It can be seen that during La Niña the maximum of the zonal wind stress anomalies is shifted further to the west by approximately $10^{\circ}$, which leads to smaller values of the area averaged zonal wind stress anomalies. Since the shift of the wind stress pattern cannot be due to a shift in the SST pattern because the SST pattern is fixed in the model, it has to be caused by different circulation patterns for warm and cold SST anomalies.

This nonlinear relationship can also be seen if one has a look at the distribution of central Pacific zonal wind stress anomalies over NINO3 SST anomalies (Fig. 2d). One can see that for large SST anomalies the linear regression does not fit to the data. For comparison also the regression curve resulting from a quadratic fit (dashed line) is shown. Especially for large SST anomalies the quadratic fit is more suitable.

To test, whether this quadratic relationship between central Pacific zonal wind stress anomalies and NINO3 SST anomalies could cause the nonlinearities in the RECHOZ model, we included this quadratic relationship in the REOSC-MC model (replacing the linear relationship) and integrated the model for 1000 years. With replacing the linear against a nonlinear relationship between central Pacific zonal wind stress anomalies and NINO3 SST anomalies, the REOSC-MC model is able to simulate the skewness and kurtosis of ENSO (Fig. 2e).

To better understand the seasonality of the RECHOZ model, the seasonal parameter values as they result from the model statistics can be analyzed. For each calendar month a separate parameter fit to the RECHOZ simulation output was performed by using a 3 month moving data block. While the damping of the temperature $a_{11}$ shows a strong seasonality the damping of the thermocline $a_{22}$ shows almost no seasonal cycle. Also the coupling parameter $a_{21}$ shows only little seasonality while the coupling parameter $a_{12}$ shows a stronger seasonal cycle (Fig. 3a and 3b). In summary the most significant 
seasonality is found in the damping of the temperature $a_{11}$. This seasonality in the RECHOZ model can only be caused by the atmospheric forcings $\tau$ and $f$.

The regressions of the forcings to the NINO3 SST (Fig. 3c) show that the coupling of the zonal wind stress is strongest in spring and early summer when the damping $a_{11}$ is weakest and the damping $a_{11}$ is strongest in autumn and winter when the coupling to the net heat flux is strongest and the coupling to the zonal wind stress is relatively weak. This is in agreement with Galanti and Tziperman [2000], who calculated the ocean-atmosphere coupling strength in a delayed-oscillator model.

The seasonal varying parameters were included in the REOSC-MC model and the model was again integrated for 10000 years. With the seasonal varying parameters included the REOSC-MC model is able to produce seasonality similar to the RECHOZ model (Fig. 3d). A large part of this seasonal cycle can be explained by the seasonality of the damping parameter $a_{11}$ only.

\section{Conclusion}

We introduced a new hybrid coupled model RECHOZ. The model reduces the oceanic part of the ENSO mode to the minimalistic low-order 2-dimensional model of Burgers et al. [2005], which by construction allows only linear and seasonally non-varying feedbacks in the ocean processes. The atmospheric part is kept to the full complexity of a high-dimensional GCM, potentially allowing nonlinear and seasonally varying feedbacks.

Although the RECHOZ model has only a minimum complex representation of ENSO, it gives a very good simulation of it. The model is able to simulate the main characteristics of ENSO like variance, seasonality, skewness, and kurtosis. The origin of these characteristics in the RECHOZ model has to lie in the atmospheric forcings. The asymmetry between eastern Pacific SST anomalies during El Niño and La Niña can be explained by a nonlinear zonal wind response to equal-strength but opposite SST anomalies, which is in agreement with the results of Kang and Kug [2002]. So the fact that this simple model with only parameterized ocean dynamics can reproduce the ENSO amplitude asymmetry shows 
that atmospheric nonlinearities have an important influence on the SST skewness in the eastern tropical Pacific.

The seasonal cycle in the strength of the ENSO amplitudes can be attributed to the seasonal varying sensitivity of the atmosphere to SST anomalies. Here it seems that the stronger coupling of zonal wind stress to SST in spring and summer reduces the damping of SST. The stronger coupling to net heat flux and weaker coupling to zonal wind stress does increase the damping of the ENSO events in winter and early spring. 


\section{Acknowledgements}

We like to thank Gerrit Burgers for very helpful comments and discussions as well as Mojib Latif and

Noel Keenlyside for helpful remarks. This work was supported by the Deutsche Forschungsgemeinschaft (DFG) through project DO1038/2-1. 


\section{References}

An, S.-I., and F.-F. Jin (2004), Nonlinearity and Asymmetry of ENSO, J. Climate, 17, 2399-2412.

Burgers, G., and D. B. Stephenson (1999), The "Normality" of El Niño, Geophys. Res. Lett., 26, 10271030.

Burgers, G., F.-F. Jin, and G. J. van Oldenborgh (2005), The simplest ENSO recharge oscillator, Geophys. Res. Lett., 32, L13706, doi:10.1029/2005GL022951.

Battisti, D. S. (1988), The dynamics and thermodynamics of a warming event in a coupled tropical atmosphere-ocean model. J. Atmos. Sci., 45,2889-2919.

Dommenget, D., and M. Latif (2008), Generation of hyper climate modes, Geophys. Res. Lett., 35, L02706, doi:10.1029/2007GL031087.

Galanti E., and E. Tziperman (2000), ENSO's Phase Locking to the Seasonal Cycle in the Fast-SST, Fast-Wave, and Mixed-Mode Regimes, J. Atmos. Sci., 57, 2936-2950.

Hirst, A. C. (1986), Unstable and damped equatorial modes in simple coupled ocean-atmosphere models. J. Atmos. Sci., 43, 606-630.

Hoerling, M. P., A. Kumar, and M. Zhong (1997), El Nino, La Nina, and the nonlinearity of their teleconnections, J. Climate, 10, 1769-1786.

Jansen, M. F., D. Dommenget, and N. Keenlyside (2009): Tropical atmosphere-ocean interactions in a conceptual framework. J. Climate, 22, 550-567

Jin, F.-F. (1997), An equatorial recharge paradigm for ENSO: Part 1: Conceptual model, J. Atmos. Sci., $54,811-829$.

Jin, F.-F., S.-I. An, A. Timmermann, and J. Zhao (2003), Strong El Niño events and nonlinear dynamical heating, Geophys. Res. Lett., 30, doi:10.1029/2002GL016356.

Kang, I.-S., and J.-S. Kug (2002), El Niño and La Niña sea surface temperature anomalies: Asymmetry characteristics associated with their wind stress anomalies, J. Geophys. Res., 107(D19), 4372, 
doi:10.1029/2001JD000393.

Philander, S. G. H. (1983), El Niño Southern Oscillation phenomena. Nature, 302, 295-301.

Philander, S. G. H. (1985), El Niño and La Niña, J. Atmos. Sci., 42, 2652-2662.

Philip, S. Y., and G. J. van Oldenborgh (2009), Significant atmospheric nonlinearities in the ENSO cycle, J. Climate, 14, 4014-4028, doi:10.1175/2009JCLI2716.1.

Rasmusson, E., and T. Carpenter (1982), Variations in tropical sea surface temperature and surface wind fields associated with the Southern Oscillation/El Niño, Mon. Wea. Rev., 110, 354-384

Rayner, N. A., D. E. Parker, E. B. Horton, C. K. Folland, L. V. Alexander, D. P. Rowell, E. C. Kent, and A. Kaplan (2003), Global analyses of sea surface temperature, sea ice, and night marine air temperature since the late nineteenth century, J. Geophys. Res., 108(D14), 4407, doi:10.1029/2002JD002670.

Roeckner, E., et al. (2003), The atmospheric general circulation model ECHAM5. Part I: Model description, Rep. 349, Max-Planck-Inst. for Meteorol., Hamburg, Germany.

Smith, N. (1995), An improved system for tropical ocean sub-surface temperature analyses, J. Atmos. Oceanic Technol., 12, 850-870

Su, J., R. Zhang, T. Li, X. Rong, J.-S. Kug, and C.-C. Hong (2010), Causes of the El Niño and La Niña amplitude asymmetry in the equatorial eastern Pacific, J. Climate, 23, 605-617, doi:10.1175/2009JCLI2894.1.

Tziperman, E., M. A. Cane, S. Zebiak, Y. Xue, and B. Blumenthal (1998), Locking of El Niño's peak time to the end of the calendar year in the delayed oscillator picture of ENSO, J. Climate, 11, 21912199. 


\section{List of Figures}

1 (a) Spectra of monthly mean NINO3 SST anomalies for RECHOZ (solid line) compared to observations (dashed) and REOSC-MC (dotted). The grey shading indicates the $80 \%$ confidence level. (b) Standard deviation of monthly mean NINO3 SST anomalies for each calendar month for RECHOZ (solid line) compared to observations (dashed) and REOSC-MC (dotted). (c) Seasonally resolved cross correlation between NINO3 SST anomalies and central Pacific thermocline depth anomalies. In the lower panels histograms of monthly mean NINO3 SST anomalies for (d) observations, (e) RECHOZ and (f) REOSC-MC.

(a) Model composite mean values of zonal wind stress anomalies for all El Niño years (T(december) $>\sigma(T))$ averaged from December to May of the following year. The black box indicates the area over which the zonal wind stress anomalies are averaged. (b) As (a) but for all La Niña years $(\mathrm{T}($ december $)<-\sigma(\mathrm{T}))$ multiplied by -1 . (c) Scatter plot of NINO3 heat flux anomalies and NINO3 SST anomalies with linear regression line. The contour interval is 0.005 $\mathrm{K} /\left(\mathrm{W} / \mathrm{m}^{2}\right)$. (d) Scatter plot of central Pacific zonal wind stress anomalies and NINO3 SST anomalies with linear regression line (solid) and the quadratic regression curve (dashed). The contour interval is $5.0 \mathrm{~K} / \mathrm{Pa}$. (e) Histogram of monthly mean NINO3 SST anomalies for REOSC-MC with the quadratic relationship included.

3 (a) Monthly fitted damping parameters $\mathrm{a}_{11}$ and $\mathrm{a}_{22}$ of RECHOZ with $95 \%$ confidence intervals.

(b) Monthly fitted coupling parameters $\mathrm{a}_{12}$ and $\mathrm{a}_{21}$ of RECHOZ with $95 \%$ confidence intervals.

(c) Monthly regression of central Pacific zonal wind stress anomalies and NINO3 SST anomalies (solid line) and of NINO3 heat flux anomalies and NINO3 SST anomalies (dashed). (d) Standard deviation of monthly mean NINO3 SST anomalies for each calendar month for 


\begin{tabular}{|c|c|c|c|}
\hline Param. & Value & Param. & Value \\
\hline \multirow{2}{*}{$a_{11}$} & $-0.076 \mathrm{month}^{-1}$ & $a_{11 O}$ & $-0.488 \mathrm{month}^{-1}$ \\
\cline { 3 - 4 } & & $a_{11 \mathrm{~A}}$ & $0.412 \mathrm{month}^{-1}$ \\
\hline$a_{12}$ & $0.021 \mathrm{~K} \mathrm{month}^{-1} \mathrm{~m}^{-1}$ & & \\
\hline$a_{21}$ & $-1.400 \mathrm{~m} \mathrm{~K}^{-1} \mathrm{month}^{-1}$ & $a_{21 O}$ & $-1.322 \mathrm{~m} \mathrm{~K}^{-1} \mathrm{month}^{-1}$ \\
\cline { 3 - 4 } & & $a_{21 \mathrm{~A}}$ & $-0.078 \mathrm{~m} \mathrm{~K}^{-1} \mathrm{month}^{-1}$ \\
\hline$a_{22}$ & $-0.008 \mathrm{month}^{-1}$ & & \\
\hline$r_{T \tau}$ & $7.32 \cdot 10^{10} \mathrm{~kg} \mathrm{~m}^{-1} \mathrm{month}^{-2} \mathrm{~K}^{-1}$ & & \\
\hline$r_{T f}$ & $1.51 \cdot 10^{20} \mathrm{~kg} \mathrm{month}^{-3} \mathrm{~K}^{-1}$ & & \\
\hline$m c$ & $1025 \mathrm{~kg} \mathrm{~m} \mathrm{~m}^{-3} \cdot 3994 \mathrm{Jg}^{-1} \mathrm{~K}^{-1} \cdot 80 \mathrm{~m}$ & & \\
\hline$c_{\tau A}$ & $5.63 \cdot 10^{-12}{\mathrm{Km} \mathrm{month} \mathrm{kg}^{-1}}$ & & \\
\hline$c_{\tau O}$ & $1.07 \cdot 10^{-13} \mathrm{~m}^{2} \mathrm{month} \mathrm{kg}^{-1}$ & & \\
\hline
\end{tabular}

Table 1: Parameter values for the recharge oscillator ocean model 
(a) zonal wind stress ano (El Nino)
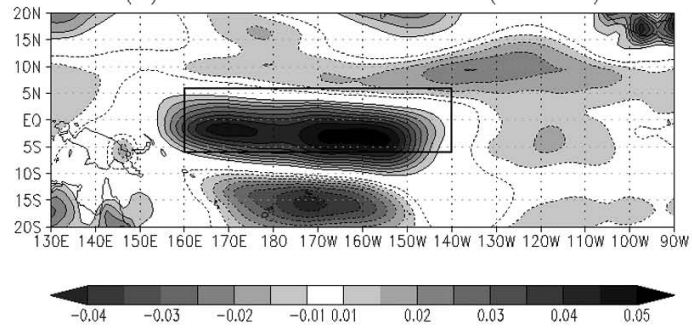

c) Regression between heat flux ano and NINO3 SSTA

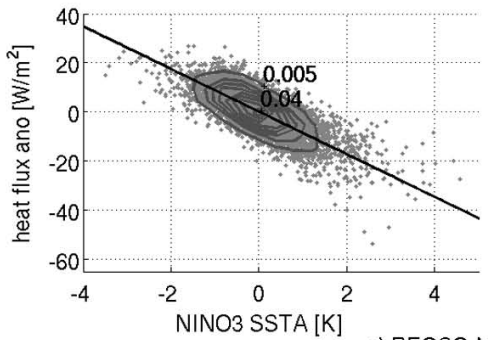

(b) -1 * zonal wind stress ano (La Nina)

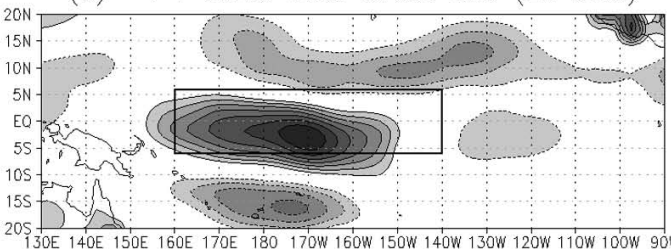

d) Regression between zonal wind stress ano and NINO3 SSTA

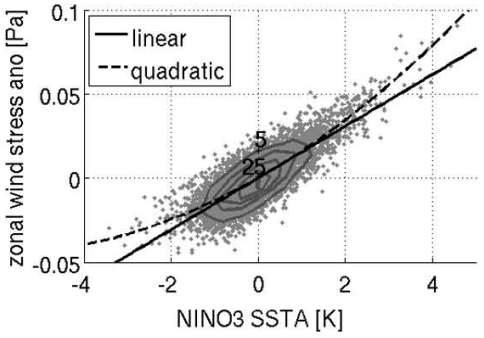

e) REOSC-MC-quadratic NINO3 SSTA histogram

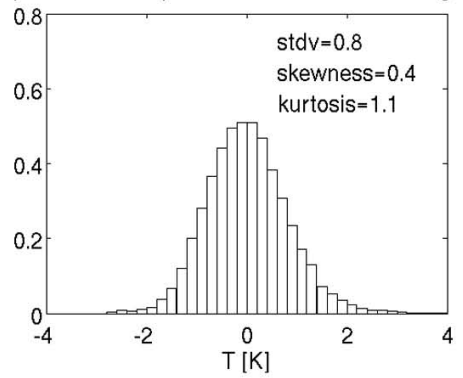


a) monthly damping parameters

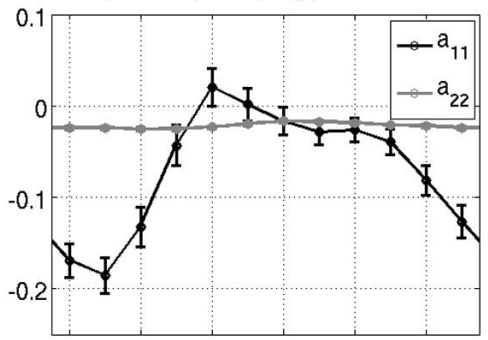

Jan Mar May Jul Sep Nov

c) monthly regression with NINO3 SSTA

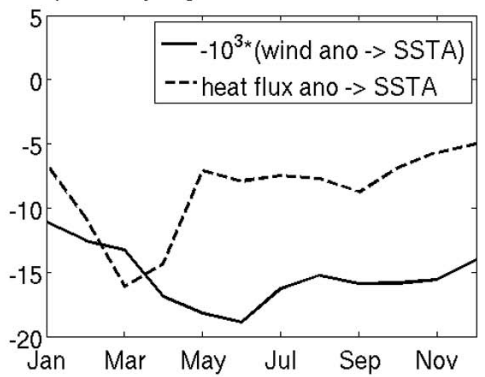

b) monthly coupling parameters
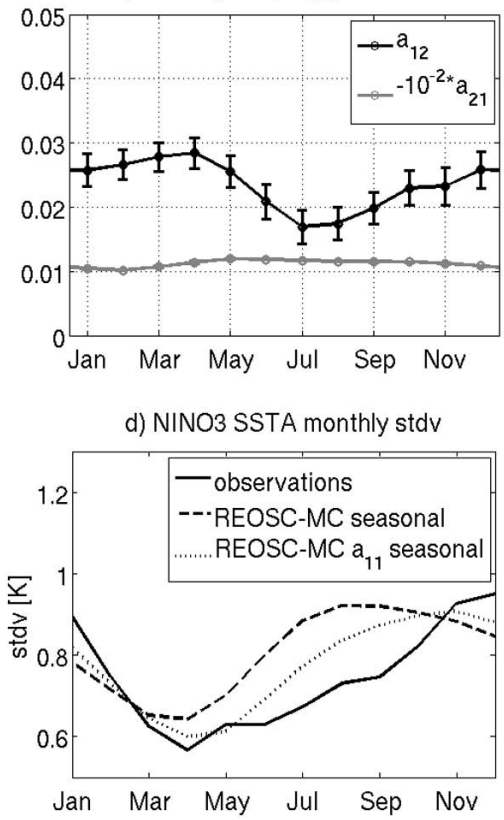